\title{
Efficient Initial Designs for Binary Response Data
}

\author{
Juha Karvanen \\ Department of Health Promotion and Chronic Disease Prevention, \\ National Public Health Institute, \\ Mannerheimintie 166, 00300 Helsinki, Finland \\ juha.karvanen@ktl.fi
}

March 2, 2019

\begin{abstract}
In this paper we introduce a binary search algorithm that efficiently finds initial maximum likelihood estimates for sequential experiments where a binary response is modeled by a continuous covariate. The problem is motivated by switching measurements on superconducting Josephson junctions. In this quantum mechanical experiment, the current is the controlled covariate and a binary response indicating the presence or the absence of a voltage response is measured. The prior knowledge on the model parameters is typically poor, which may cause the common approaches of initial estimation to fail. The binary search algorithm is designed to work reliably even when the prior information is very poor. The properties of the algorithm are studied in simulations and an advantage over the initial estimation by equally spaced covariates is demonstrated. We also study the cost-efficiency of the binary search algorithm and find the approximately optimal number of measurements per stage when there is a cost related to the number of stages in the experiment.
\end{abstract}

KEY WORDS: optimal design, binary search, logistic regression, complementary log-log, quantum physics, switching measurement

\section{Introduction}

Consider an experiment where the probability of success (or failure) is a monotonic function of a controlled covariate. The functional form of the response curve is known but the parameters defining its location and slope are unknown. Our goal is to estimate these unknown parameters but the problem is that our prior knowledge on the parameters is very poor. Intuitively it is clear that we should choose such covariate values that both successes and failures are measured as responses - otherwise the experiment provides very little information 
on the parameters of the interest. But if the location and slope of the response curve are unknown, we do not known the optimal covariate values.

The problem described above is encountered in a quantum mechanical experiments called switching measurements (Josephson, 1962; Grabert and Devoret, Eds.; Pekola et al., 2005). In the experiment, a Josephson junction circuit is placed in a dilution refrigerator at temperature below 4.2 kelvin. The height of a current pulse measured in nanoamperes is the controlled covariate and the absence or presence of a voltage pulse is measured as a response. For a certain narrow range of current pulses, the presence of the voltage response is a random variable depending on the height of the current pulse. This allows the Josephson junctions to be used as as ultra-sensitive sensors. The problem is that we do not know the sensitive range in advance. Only imprecise limits can be given: the current must above zero and a rough upper limit is obtained by measuring the resistance of the circuit in room temperature.

In the literature on the optimal design for binary response models, it is often assumed that prior estimates are readily available or that they can easily achieved from a pilot experiment. A natural but not always very efficient approach to initial estimation is to choose the initial covariate values equally spaced on some initial range. This approach has been employed in several theoretical and practical works, e.g. (Sitter, 1992; Sitter and Wu, 1999; Chaudhuri and Mvkland, 1993; Chaloner and Verdinelli, 1995). A more advanced approach was proposed in (Sitter, 1992; King and Wong, 2000) where the problem of selecting the initial design was studied using the minimax principle. All these approaches may fail if the prior information is very poor.

In this paper we study the problem of initial design in detail and introduce a binary search algorithm that reliably finds initial maximum likelihood estimates (MLEs) even in the case where the prior information on the parameters is very poor. The binary search algorithm was already successfully applied to switching measurements (Karvanen et al., 2005). We also study the cost-efficiency of the binary search algorithm and find the approximately optimal number of measurements per stage assuming that there a cost related to the number of stages in the experiment.

The rest of the paper is organized as follows. In Section 2 we review some commonly used binary response models and present the related theory of optimal design. The problem of the non-existence of MLEs with small and moderate sample sizes is studied. In Section 3 the binary search algorithm is introduced and its properties are studied. In Section 4 the binary search algorithm is compared to the initial estimation with equally spaced covariates. In Section [5] we consider a cost model where there is a cost related to the number of measurements and a cost related to the number of stages and find the approximately optimal number of measurements in the binary search. In Section [6 we apply the results to switching measurements and discuss the use of binary search in applications similar to the sport fishing example (Sitter, 1992; King and Wong, 2000). Section 7 concludes the paper. 


\section{Binary response models}

There are three parametric models, the logit, the probit and the complementary $\log -\log (\operatorname{clog} \log )$ model, that are frequently used to model the dependence between the binary response $Y$ and the continuous covariate $x$. All these models can be presented in the framework of generalized linear models (McCullagh and Nelder, 1989)

$$
P(Y=1)=\mathrm{E}(Y)=F(a x+b),
$$

where the response curve $F$ is a cumulative distribution function (cdf) and the $a$ and $b$ are the parameters of the model to be estimated. The three response curves commonly used are: logistic distribution for the logit model

$$
F(a x+b)=\frac{\exp (a x+b)}{1+\exp (a x+b)},
$$

normal distribution for the probit model

$$
F(a x+b)=\Phi(a x+b)=\int_{-\infty}^{\infty} \frac{1}{\sqrt{2 \pi}} \exp \left(\frac{-(a x+b)^{2}}{2}\right) \mathrm{d} x
$$

and the Gompertz distribution for the cloglog model

$$
F(a x+b)=1-\exp (-\exp (a x+b)) .
$$

The theory of optimal design provides results how the covariate values should be chosen in order to estimate parameters $a$ and $b$ optimally. Table 1 adapted from (Ford et al., 1992) presents the (locally) D-optimal covariate values for the logit, the probit and the cloglog model. The apparent problem discussed in more detail by Minkin (1987) and Sitter (1992) is that the optimal covariate values are functions of the unknown parameters that we should estimate. It follows that we can design the optimal experiment only if we already know the parameters we want estimate, which is an impractical requirement. The problem is often evaded assuming that we already have good initial estimates. Less often it is discussed how the good initial estimates are obtained and what follows if initial information is very poor.

Next we study the problem of the non-existence of MLEs. For this we assume that we know the true values of $a$ and $b$ and can therefore use the actual (locally) D-optimal design. We consider maximum likelihood estimation in small experiments where the sample size $n \leq 200$. MLEs cannot be estimated from the measured data if it is possible to classify the responses to 1's and 0's on the basis of the covariate values, e.g. response 1 is obtained if $x>100$ and response 0 is obtained if $x<100$. More formally, the existence of MLEs for the model (1) requires that (Albert and Anderson, 1984)

$$
\begin{aligned}
& \max \left(x_{i} \mid y_{i}=0\right)>\min \left(x_{i} \mid y_{i}=1\right) \text { and } \\
& \max \left(x_{i} \mid y_{i}=1\right)>\min \left(x_{i} \mid y_{i}=0\right),
\end{aligned}
$$


Table 1: D-optimal covariate values for the models (2), (3) and (4). The Doptimal covariate values $x_{1}^{*}$ and $x_{2}^{*}$ are solved from equations $a x_{1}^{*}+b=z_{1}^{*}$ and $a x_{2}^{*}+b=z_{2}^{*}$. Columns $F\left(z_{1}^{*}\right)$ and $F\left(z_{2}^{*}\right)$ report the cdf values related to the optimal covariates, i.e., the probabilities of response 1 .

\begin{tabular}{rcccc} 
model & \multicolumn{4}{c}{ D-optimal covariates } \\
& $z_{1}^{*}$ & $z_{2}^{*}$ & $F\left(z_{1}^{*}\right)$ & $F\left(z_{2}^{*}\right)$ \\
\hline $\operatorname{logit}$ & -1.543 & 1.543 & 0.176 & 0.824 \\
probit & -1.138 & 1.138 & 0.128 & 0.872 \\
cloglog & -1.338 & 0.980 & 0.231 & 0.930
\end{tabular}

where $y_{i}$ is the measured response for the covariate value $x_{i}$. This condition is fulfilled, for instance, if we have two covariate values in such a way that for each of them both 0's and 1's are measured as responses. It follows that the probability of MLE non-existence under the D-optimal design is equivalent to the probability the only 0's or 1's are measured as responses in either at $z_{1}^{*}$ or at $z_{2}^{*}$. According to the elementary laws of probability calculus, this probability can be expressed as

$$
P(\text { No MLE })=q_{1}^{s}+p_{1}^{s}+q_{2}^{s}+p_{2}^{s}-q_{1}^{s} q_{2}^{s}-q_{1}^{s} p_{2}^{s}-p_{1}^{s} q_{2}^{s}-p_{1}^{s} p_{2}^{s},
$$

where $p_{1}=F\left(z_{1}^{*}\right)$ and $p_{2}=F\left(z_{2}^{*}\right)$ from Table \ $q_{1}=1-p_{1}, q_{2}=1-p_{2}$, $s=n / 2$ and $n$ is the sample size, which is assumed to be an even number. The probabilities of MLE non-existence in D-optimal designs as a function of sample size are presented in Table 2 It can be seen that surprising large sample sizes are needed if we want to avoid the risk that MLEs cannot be calculated from the sample. The conclusion here is that even if we are using the D-optimal design, the non-existence of MLE is a problem that cannot be ignored when the sample size is small. In practical situations where the D-optimal design is not known the problem may be even more serious.

Table 2: Probabilities for the non-existence of MLEs when the D-optimal covariate values from Table 1 are used.

\begin{tabular}{rccc} 
sample size & logit & probit & cloglog \\
\hline 4 & 0.91584142 & 0.95047339 & 0.95394709 \\
10 & 0.61553275 & 0.75551315 & 0.77862340 \\
20 & 0.26765833 & 0.44578006 & 0.52280966 \\
40 & 0.04117204 & 0.12633768 & 0.23974289 \\
100 & 0.00012482 & 0.00217817 & 0.02698092 \\
200 & 0.00000001 & 0.00000237 & 0.00072786
\end{tabular}




\section{Initial estimation with binary search}

We start our introduction to the binary search algorithm considering types of the prior information. There is virtually always some prior information on the parameters available although this prior information may be very inaccurate and difficult to express in mathematical form. For instance, the following types of prior information may be encountered:

- Point estimates of the parameters from the previous study/studies.

- Range of the possible parameter values.

- Prior distribution of the parameters.

- Range of sensible covariate values.

We concentrate to prior information given as a range of covariate values. This is the simplest type of prior information and the other types can be transformed to this type if needed. It is actually difficult to find a practical situation where we could not specify any initial interval for the covariate. Value zero is often a theoretical lower bound for the covariate and some extreme high value can serve as the upper bound. In the other words, we can always specify the initial interval such a way that it contains all sensible covariate values with high probability. The initial interval can become very wide if the prior information is very inaccurate but this is not a problem to the binary search algorithm.

The key idea of the binary search algorithm is to measure at such covariate values that the condition for MLE existence (5) is fulfilled as quickly as possible. The motivation for this is that after the MLEs have been found, we can continue the experiment using the design that is optimal for the current estimates of the parameters. The proposed method is similar to the binary search algorithm that finds a root of a continuous function. Instead of a root we are looking for a covariate value where we can measure both 0's and 1's as responses. At every step we measure the responses at the middle point of the current search interval. If only 0's are measured, the middle point is taken as the new starting point of the interval. If only 1's are measured, the middle point is taken as the new end point of the interval. The first part of the binary search ends when both 0's and 1's are measured at same point $x$. Another covariate value needed to guarantee the MLE existence is then found in the neighborhood of $x$. The proposed algorithm can be presented as follows:

1. Use the previous knowledge to construct such an interval $\left[x_{\min }, x_{\max }\right]$ that we can be sure that $F\left(x_{\max } ; a, b\right)-F\left(x_{\min } ; a, b\right)$ is close to 1 . Constructing this kind of interval is usually possible even if we have very little knowledge on the parameters.

2. Use binary search to find such a point $x$ from the interval $\left[x_{\min }, x_{\max }\right]$ that both 0's and 1's are measured as responses. In binary search, we measure the responses at the middle point of the current interval. If only 0's are 
measured, the middle point is taken as the new starting point of the interval. If only 1's are measured, the middle point is taken as the new end point of the interval. Let $x$ be found as the middle point of the interval $\left[x_{l}, x_{u}\right]$, i.e. $x=\left(x_{l}+x_{u}\right) / 2$.

3. Define $|\epsilon|=\left(x_{u}-x_{l}\right) / 4$. The sign of $\epsilon$ is determined according to the measured response: $\operatorname{sign}(\epsilon)=\operatorname{sign}(0.5-\bar{y})$, where $\bar{y}$ is the average response for $x$. If $0.5-\bar{y}=0$ the sign of $\epsilon$ is chosen randomly.

4. Measure first at point $x+\epsilon$. If both 0's and 1's were not measured as responses, measure also at point $x-\epsilon$.

5. If MLEs exist for the data measured so far, proceed to the maximum likelihood estimation. Otherwise, divide $\epsilon$ by two and return to Step 4 .

An illustration of the progress of the algorithm is presented in Figure 1 Step 2 requires that we measure at least two times at each point. The optimal number of measurements is determined in Section [5] When the binary search in Step 2 converges we have found one of the two points required for the maximum likelihood estimation. The other point that is needed is then found in the neighborhood of point $x$ using again binary search. Due to use of binary searches, the procedure works reliably and efficiently. At every step of binary search, the length of the current interval is divided by two. Consequently, the procedure converges exponentially fast regardless of the choice of the initial interval.

After it has been found that condition (5) holds and the MLEs therefore exist, there are two alternative ways to use the data. We may calculate the MLEs from all data that has been cumulated in the binary search algorithm (MLE method I) or use only the endpoints of the initial interval and the two points found in Steps 2 and 4 of the algorithm (MLE method II). Neither of these approaches is fully satisfactory because the data origin from a procedure with data dependent stopping rules. However, the purpose of the initial design is only to produce some initial estimates for the primary experiment and in this sense any sensible estimate based on real data is better than an arbitrary guess.

The performance of the proposed binary search algorithm is studied in simulations where the quality of prior information varies from poor to very poor. We fix the parameters $a=1$ and $b=0$ and specify the initial interval for covariate values as $[h-d, h]$, where $d>2 h$ is the length of the initial interval (a simulation parameter) and $h$ is generated from Uniform $(4, d-4)$ distribution. The results can be generalized because arbitrary parameters $a$ and $b$ can be returned to the canonical case $a=1$ and $b=0$ by a linear transformation. Note that the initial intervals are specified in such way that the middle point of the response curve always belongs to the interval but its relative location respect to the endpoints of the interval is unknown and uniformly distributed. The interval lengths $d \in\{10,15,20,50,100,200,1000\}$ are used in the simulations. The number of measurements per stage $n_{k}$ is also a simulation parameter and has 23 values $\{2,3,4, \ldots, 200,500,1000\}$. 5000 simulation runs are performed for each combination of simulation parameters. For each simulation run we record 


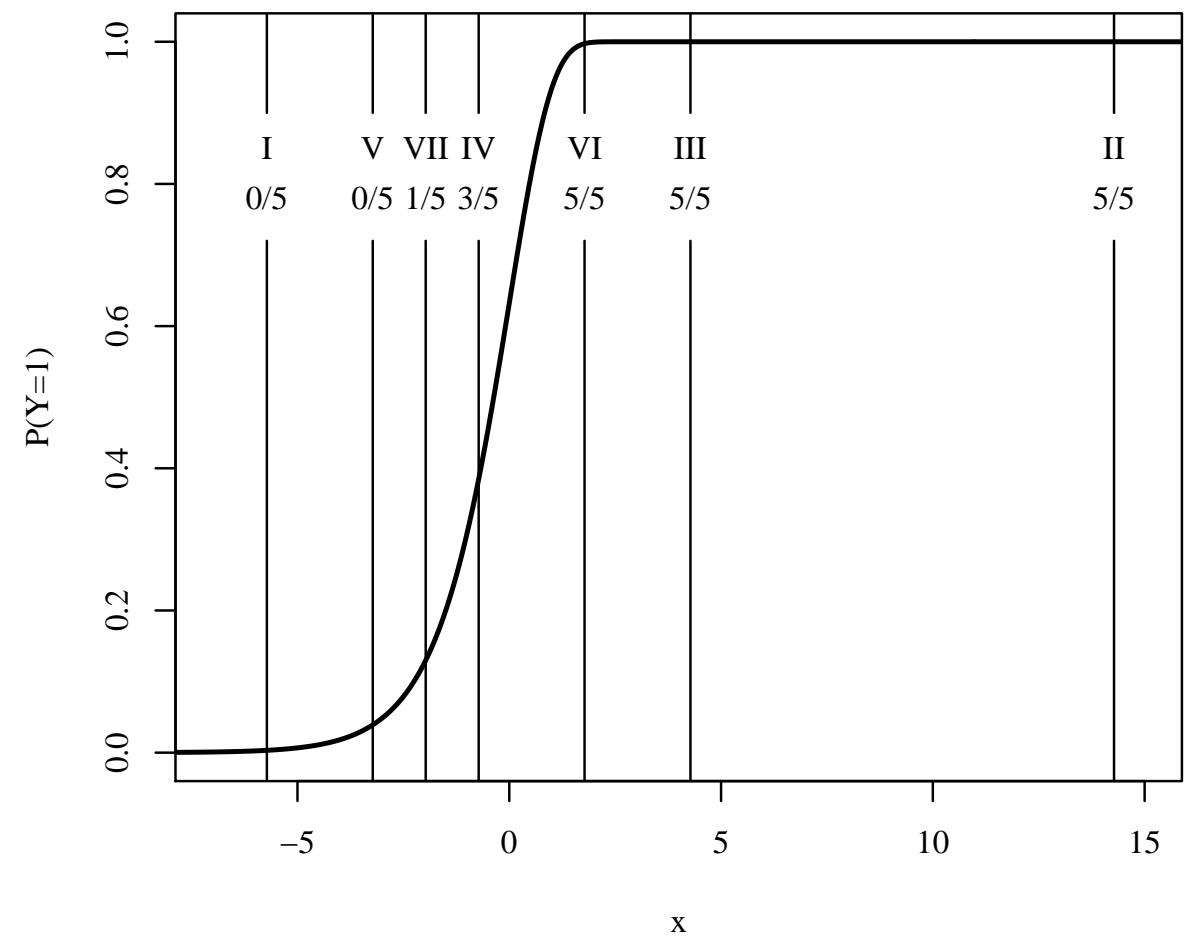

Figure 1: Binary search algorithm in action. The initial interval was defined as $[-5.7,14.3]$. The vertical lines present the measured covariate values and the Roman numerals indicate the order of the measurements. The numbers below the Roman numerals report the number of responses 1 per the total measurements; e.g. 3/5 means that three responses 1 were measured out of five measurements. The obtained initial estimates of the parameters of the cloglog model and their standard errors are $\hat{a}=1.83(0.88)$ and $\hat{b}=1.88(1.29)$. 
- $K$, the number of stages needed for the convergence of the binary search,

- $\mathbf{J}_{K}$, the Fisher information matrix after the initial design,

- $\hat{a}$ and $\hat{b}$, the parameter estimates after the initial design.

The validity of the initial interval is always first checked measuring the responses at the ends of the initial interval. These measurements are counted as the two first stages and the actual binary search starts from the stage three.

Tables [3 4 and 5] report the simulation results for the cloglog model. It can be seen from Table 3 that more stages are needed when the initial interval widened but the number of the required additional stages is small. In fact, if the length of the initial interval is multiplied by two, one additional stage is required because at each stage of the binary search algorithm, the current interval is halved. It follows that if the length of the initial interval is multiplied by ten, $\log _{2}(10) \approx 3.32$ additional stages are required, which is in the agreement with the differences between the observed $K$ for $d=10, d=100$, and $d=1000$ in Table 3

The estimates using the MLE method I and the MLE method II are presented in Tables 4 and 5 respectively. The averages of estimates $\hat{a}$ and $\hat{b}$ indicate that $a$ and $b$ are systemically overestimated with the method I. The bias is large for small stage sizes (average $\hat{a}=2.56$ for $n_{k}=3$ ) and small for large stage sizes $\left(\hat{a}=1.04\right.$ for $\left.n_{k}=100\right)$ but seems independent of the length of the initial interval $d$. For $n_{k}=2$, the average bias is very large indicating that some estimates were very large. The reason for the bias is that there is only limited number of outcomes when the initial estimation may stop. With method II, $a$ is underestimated when $n_{k}=2$. On the basis of these results, it is clear that the stage size $n_{k}=2$ should not be used. With method II, parameter $a$ is slightly overestimated for $n_{k}>2$ when $d=10$ and underestimated when $d=100$ and $d=1000$. Parameter $b$ is slightly underestimated. Similarly to method I, the bias decreases when $n_{k}$ increases.

There are several ways to deal with the bias. The safest way is to choose large $n_{k}$. If small $n_{k}$ is preferred, the results in Tables 4 and 5 could be employed in making $\hat{a}$ and $\hat{b}$ unbiased but this is not necessarily a good solution for method I because the distribution of $\hat{a}$ is strongly skewed, which can be seen comparing the mean and the 95th percentile. Simpler approach is to use the estimates without a bias correction and rely that the following sequential design will quickly correct the bias. Overestimated $a$ causes the D-optimal covariate values calculated according to Table 1 to shift towards to the center of the response curve. This is not particularly harmful because both 0's and 1's can be measured as responses in the central area of the response curve.

The Fisher information after convergence of the binary search is almost independent of the length of the initial interval. This can be explained by the fact that when the initial interval is wide, the measurement points at the first stages fall to the tails of the response curve and thus provide very little information in terms of the Fisher information. The number of stages that 
provide the information is therefore nearly constant. The same fact explains why the Fisher information of method I and method II are almost the same.

Table 3: Simulation results for the cloglog model using the binary search algorithm. The numbers reported are mean, 5th percentile and 95th percentile calculated from 5000 simulation runs for different lengths of the initial interval $d$ and number of measurements per stage $n_{k}$.

\begin{tabular}{|c|c|c|c|c|c|c|c|c|c|}
\hline \multicolumn{10}{|c|}{$K$, the number of stages needed for the convergence } \\
\hline & \multicolumn{3}{|c|}{$d=10$} & \multicolumn{3}{|c|}{$d=100$} & \multicolumn{3}{|c|}{$d=1000$} \\
\hline$n_{k}$ & mean & $5 \%$ & $95 \%$ & mean & $5 \%$ & $95 \%$ & mean & $5 \%$ & 95 \\
\hline 2 & 7.53 & 5 & 11 & 10.93 & 8 & 14 & 14.26 & 12 & \\
\hline 3 & 6.53 & 4 & 9 & 9.87 & 8 & 13 & 13.27 & 11 & \\
\hline 4 & 6.09 & 4 & 8 & 9.52 & 8 & 12 & 12.93 & 11 & \\
\hline 5 & 5.81 & 4 & 8 & 9.25 & 7 & 12 & 12.69 & 11 & \\
\hline 10 & 5.11 & 4 & 7 & 8.72 & 7 & 11 & 12.19 & 10 & \\
\hline 100 & 4.25 & 4 & 5 & 7.83 & 6 & 10 & 11.35 & 10 & \\
\hline 1000 & 4.09 & 4 & 5 & 7.28 & 6 & 9 & 10.91 & 9 & \\
\hline
\end{tabular}

Full simulation results including results for the logit model and the probit model can be obtained from the author by a request. The results for the logit model and the probit model are otherwise similar to the results for the cloglog model but the estimates of $b$ are unbiased because of the symmetry of the response curves.

\section{Comparison to equally spaced covariates}

A commonly used practice for initial design is to choose covariate points that are equally spaced in the design space. Typically, the number of covariate points is selected on ad-hoc basis. More systematic approaches were proposed by Sitter (1992) and King and Wong (2000) who selected the initial design using the minimax principle. It was found by them that the number of initial covariate points should be increased when the uncertainty about the model parameters increases. However, when the initial range is wide and the number of initial covariate points is large, most of the covariate points will lie far from the D-optimal points and thus provide very little information on the parameters of the interest. When the prior information is very poor, there is a substantial risk that the equal spacing will just spend resources without any guarantee of finding MLEs. We study the performance of initial estimation with equally spaced covariates in the simulation setting presented in Section 3 The additional simulation parameters are the number of covariate points $M$ and the number measurements per each covariate point $n_{m}$. $M$ has values $5,10,50,100$ and 1000 and $n_{m}$ has some suitably chosen values that lead to a reasonable sample size $M n_{m}$. For each combination of $d, M$ and $n_{m}, 10000$ simulation runs are performed and the existence of MLEs is checked using the condition (5). 
Tables 6 reports the empirical probabilities of MLE non-existence for the cloglog models. The results show a dramatic change in the performance when the quality of the prior information decreases. For instance, if $d=10$, the strategy with $M=10$ and $n_{m}=10$ finds MLEs practically always but if $d=100$ the same strategy fails almost without exceptions. If $d=1000$, the existence of MLEs cannot be guaranteed even if $M=1000$ and $n_{m}=2$. In general it seems that if the sample size $M n_{m}$ is fixed, it is a better have large $M$ and small $n_{m}$ than vice versa. This is in agreement with the results by Sitter (1992) and King and Wong (2000).

Initial estimation with equally spaced covariates cannot compete with the binary search approach. Comparing Tables 3 and [6] we see that the required total sample sizes are significantly smaller in the binary search. By looking the row $n_{k}=5$ in Table 3 we notice that the sample size of $90(=5 \cdot 16)$ was sufficient even when $d=1000$. In Table [ not even the sample size of $2000(=1000 \cdot 2)$ was sufficient to eliminate the risk of MLE non-existence.

\section{Cost-efficient initial designs}

In this section we calculate cost-efficient stage sizes for the proposed binary search procedure. We assume that there is a cost related to the number of stages and a cost related to the number of observations. Without loss of generality, we fix the marginal cost of making one additional measurement to be one and denote the cost of having one additional stage as $C_{S}$. The same cost scheme was used for D-optimal sequential design in (Karvanen, 2006). Our goal is to find the optimal number measurements for initial estimation with the binary search algorithm as a function of the stage $\operatorname{cost} C_{S}$ and the length of the initial interval $d$. Optimality is defined here as the cost needed for finding the initial MLEs. We restrict only to initial designs were the number of measurements is same for each stage of the binary search. If initial estimation with stage size $n_{k}$ takes $K$ stages, the total cost becomes

$$
C\left(n_{k}\right)=K C_{S}+K n_{k} .
$$

When the stage cost $C_{S}$ is fixed we can calculate the optimal stage size $n_{k}$ among the the stage sizes used in the simulation in Section 3. We let $C_{S}$ have 39 values from 0.001 to 1000 and calculated $n_{k}$. Based on these data, we may build the following model for the the optimal $n_{k}$

$$
\log \left(n_{k}\right)=\alpha+\beta \log d+\gamma \log C_{S},
$$

where the estimated parameters $\alpha, \beta$, and $\gamma$ for the logit, the probit and the cloglog model are presented in Table $\mathbf{7}$ In practical experiments, $d$ is always unknown a priori and $C_{S}$ may be known or unknown. We recommend that the optimal $n_{k}$ is calculated for several combinations of $d$ and $C_{S}$ using equation (8). This information may help in choosing the value of $n_{k}$. 


\section{Applications}

We reconsider the measurement data from (Karvanen et al., 2005) in order to illustrate the application of the presented approach. In the experiment, a sample consisting of aluminium-aluminium oxide-aluminium Josephson junction circuit in a dilution refrigerator at 20 millikelvin temperature was connected to computer controlled measurement electronics in order to apply the current pulses and record the resulting voltage pulses. The resistance of the sample at room temperature suggested that a pulse of $300 \mathrm{nA}$ always causes a switching (response 1). This gave the upper limit for the initial estimation. The lower limit for the initial estimation, $200 \mathrm{nA}$ was roughly estimated from the dimensions of the Josephson junction by an experienced physicist. Alternatively, $0 \mathrm{nA}$ may be used as the lower limit for the initial estimation. The initial estimation used the stage size 50. A major difference compared to algorithm presented in Section 3 was that the starting value of $\epsilon$ was set as one. In this particular situation this was a good choice because the MLEs were found after measuring only at four points. Taking the final estimates $\hat{a}=0.240$ and $\hat{b}=-60.628$ calculated from 117288 data points as the true parameters, we study the experiment in the light of the simulation results in Sections 3 and 5 The standardized length of the initial interval is $d=0.240 \cdot(300-200)=24$, or $d=0.240 \cdot 300=72$ for the wider interval. The standardized stage $\operatorname{cost} C_{S}$ was estimated from the data and value $C_{S}=228.4$ was obtained (Karvanen, 2006). The model (8) gives the optimal $n_{k}=\exp (-1.063-0.214 \log (24)+0.85 \log (228.4)) \approx 18$ for the initial interval $[200,300]$, and $n_{k} \approx 14$ for the initial interval $[0,300]$. According to Table 3 we expect that the number of stages needed is between 4 and 11 .

In addition to switching measurement, the binary search algorithm may be useful also in some other problems. Sitter (1992) and King and Wong (2000) consider an application where the problem was to evaluate the economic value of the sport fishing in British Columbia tidal waters. Fishermen were asked the question "If the cost of your fishing trip had been $x$ dollars higher today, would you still have gone fishing?" Originally, $x$ varied from $\$ 1$ to $\$ 50$ but in the middle of the survey it was found that a number of fishermen were willing to pay even more than $\$ 50$ extra and the range of $x$ was widened to cover values from $\$ 1$ to $\$ 100$. Obviously, the survey was not designed optimally. As an improvement, Sitter (1992) proposed a robust seven point design with equal weights for this problem and King and Wong (2000) improved Sitter's results by proposing a robust nine point design with unequal weights. This and similar surveys could benefit from sequential designs with efficient initial design. By using a cellular phone the interviewer could have a direct contact to the computer server collecting the data, which would allow the use of the binary search algorithm described in this paper. In this scenario, the interviewer sends the response of a fisherman to the server that after short calculations returns the amount of dollars for the next question. This would minimize the loss of efficiency due to badly chosen design space. 


\section{Conclusion}

When the problem of optimal design in binary response experiments is considered in statistical literature it is often assumed that the initial estimates are available. In this paper we propose an approach that efficiently finds these initial estimates. The approach uses binary search algorithm that quickly and reliably returns initial MLEs. After that the experiment may continue with likelihood based sequential estimation.

Results provided in the paper consider the logit, the probit and the cloglog model but the proposed approach is not restricted to any particular parametric model. On the other hand, the proposed approach relies on the idea of sequential design. If there is a considerable delay between choosing the covariate value and measuring the response, sequential designs may be impractical. This applies to many biomedical dose-response trials. In contrast, in many physical experiments the response is measured almost instantly but the total time available for the experiment may be restricted. Efficient sequential designs may be very useful in these problems.

The presented simulations compared the binary search algorithm to the initial estimation with equally spaced covariates. The key difference between the approaches is that with equally spaced covariates we should have a good guess for the initial interval whereas with the binary search algorithm any initial interval that contains the middle point of the response curve will do. If the prior information is very poor, the proposed binary search approach has a huge advan-

tage over the initial estimation by equally spaced covariates. The importance of reliable initial estimation is emphasized by the fact that if the prior information is poor it is often difficult to deduce how poor it exactly is.

\section{Acknowledgements}

The author thanks Dr. Juha J. Vartiainen for useful comments.

\section{References}

Albert, A. and Anderson, J. A. (1984). On the existence of maximum likelihood estimates in logistic regression models. Biometrika, 71:1-10.

Chaloner, K. and Verdinelli, I. (1995). Bayesian experimental design: a review. Statistical Science, 10(3):273-304.

Chaudhuri, P. and Mykland, P. A. (1993). Nonlinear experiments: optimal design and inference based on likelihood. Journal of American Statistical Association, 88(422):538-546.

Ford, I., Torsney, B., and Wu, C. F. J. (1992). The use of a canonical form in the construction of locally optimal designs for non-linear problems. Journal of the Royal Statistical Society: Series B (Statistical Methodology), 54(2):569-583. 
Grabert, H. and Devoret (Eds.), M. H. (1992). Single Charge Tunneling, volume 294 of NATO ASI Series B. Plenum, New York.

Josephson, B. D. (1962). Possible new effects in superconductive tunnelling. Physics Letters, 1:251.

Karvanen, J. (2006). Cost-efficient sequential designs for binary response models. Submitted for publication, preprint available at http://arxiv.org/pdf/math.ST/0610578

Karvanen, J., Vartiainen, J. J., Timofeev, A., and Pekola, J. (2005). Experimental designs for binary data in switching measurements on superconducting Josephson junctions. Submitted for publication, preprint available at http://arxiv.org/pdf/cond-mat/0610507

King, J. and Wong, W.-K. (2000). Minimax D-optimal designs for the logistic model. Biometrics, 56:1263-1267.

McCullagh, P. and Nelder, J. (1989). Generalized Linear Models. Chapman and Hall, London.

Minkin, S. (1987). Optimal designs for binary data. Journal of the American Statistical Association, 82(400):1098-1103.

Pekola, J. P., Nieminen, T. E., Meschke, M., Kivioja, J. M., Niskanen, A. O., and Vartiainen, J. J. (2005). Shot-noise-driven escape in hysteretic Josephson junctions. Physical Review Letters, 95(19):197004.

Sitter, R. R. (1992). Robust designs for binary data. Biometrics, 48:1145-1155.

Sitter, R. R. and Wu, C. F. J. (1999). Two-stage design of quantal response studies. Biometrics, 55:396-402. 
Table 4: Simulation results for the cloglog model using the binary search algorithm and MLE method I. The numbers reported are mean, 5th percentile and 95th percentile calculated from 5000 simulation runs for different lengths of the initial interval $d$ and number of measurements per stage $n_{k}$.

$\hat{a}$, estimated slope parameter $(a=1)$

\begin{tabular}{|c|c|c|c|c|c|c|c|c|c|}
\hline \multirow[b]{2}{*}{$n_{k}$} & \multicolumn{3}{|c|}{$d=10$} & \multicolumn{3}{|c|}{$d=100$} & \multicolumn{3}{|c|}{$d=1000$} \\
\hline & mean & 5 th & $95 \mathrm{th}$ & mean & 5 th & 95 th & mean & 5 th & 95th \\
\hline 2 & 199.58 & 0.50 & 28.79 & 221.84 & 0.64 & 27.20 & 23.22 & 0.58 & 33.49 \\
\hline 3 & 2.56 & 0.60 & 5.11 & 21.03 & 0.50 & 7.17 & 8.30 & 0.64 & 5.86 \\
\hline 4 & 1.69 & 0.57 & 3.98 & 1.64 & 0.55 & 3.64 & 1.65 & 0.58 & 3.56 \\
\hline 5 & 1.47 & 0.63 & 3.00 & 1.46 & 0.57 & 2.85 & 1.46 & 0.57 & 2.95 \\
\hline 10 & 1.20 & 0.67 & 2.07 & 1.19 & 0.68 & 2.03 & 1.19 & 0.61 & 2.05 \\
\hline 100 & 1.04 & 0.85 & 1.29 & 1.02 & 0.78 & 1.27 & 1.01 & 0.79 & 1.25 \\
\hline 1000 & 1.00 & 0.94 & 1.07 & 0.99 & 0.83 & 1.11 & 1.00 & 0.86 & 1.15 \\
\hline \multicolumn{10}{|c|}{$\hat{b}$, estimated location parameter $(b=0)$} \\
\hline$n_{k}$ & mean & 5 th & $95 \mathrm{th}$ & mean & 5 th & $95 \mathrm{th}$ & mean & 5 th & 95 th \\
\hline 3 & 0.67 & -1.05 & 2.76 & 40.77 & -1.05 & 2.74 & 14.72 & -1.01 & 2.69 \\
\hline 4 & 0.27 & -0.89 & 1.78 & 0.29 & -0.85 & 1.83 & 0.26 & -0.83 & 1.76 \\
\hline 5 & 0.20 & -0.76 & 1.47 & 0.20 & -0.75 & 1.49 & 0.19 & -0.76 & 1.46 \\
\hline 10 & 0.09 & -0.56 & 0.92 & 0.08 & -0.58 & 0.86 & 0.08 & -0.57 & 0.86 \\
\hline 100 & 0.02 & -0.21 & 0.26 & 0.01 & -0.26 & 0.29 & -0.02 & -0.30 & 0.25 \\
\hline 1000 & 0.00 & -0.07 & 0.07 & -0.03 & -0.26 & 0.11 & 0.01 & -0.15 & 0.22 \\
\hline
\end{tabular}

$D$, the square root of the determinant of the Fisher information

\begin{tabular}{rrrrrrrrrr} 
& \multicolumn{3}{c}{$d=10$} & \multicolumn{3}{c}{$d=100$} & \multicolumn{3}{c}{$d=1000$} \\
$n_{k}$ & mean & 5 th & 95 th & mean & 5 th & 95 th & mean & 5 th & 95 th \\
2 & 1.73 & 0.08 & 4.96 & 1.66 & 0.09 & 3.75 & 1.64 & 0.07 & 4.37 \\
3 & 2.69 & 0.51 & 6.44 & 2.66 & 0.51 & 6.79 & 2.73 & 0.55 & 6.09 \\
4 & 3.76 & 1.08 & 7.60 & 3.61 & 1.10 & 7.60 & 3.67 & 0.94 & 8.24 \\
5 & 4.56 & 1.30 & 9.45 & 4.46 & 1.52 & 9.13 & 4.53 & 1.51 & 9.52 \\
10 & 8.33 & 3.25 & 15.21 & 8.35 & 3.95 & 14.76 & 8.34 & 3.80 & 15.03 \\
100 & 68.58 & 30.98 & 101.87 & 55.56 & 20.57 & 89.39 & 55.60 & 24.47 & 89.33 \\
1000 & 679.14 & 425.46 & 952.25 & 370.71 & 103.53 & 756.21 & 323.80 & 86.97 & 658.73
\end{tabular}


Table 5: Simulation results for the cloglog model using the binary search algorithm and MLE method II. The numbers reported are mean, 5th percentile and 95th percentile calculated from 5000 simulation runs for different lengths of the initial interval $d$ and number of measurements per stage $n_{k}$.

$\hat{a}$, estimated slope parameter $(a=1)$

\begin{tabular}{|c|c|c|c|c|c|c|c|c|c|}
\hline \multirow[b]{2}{*}{$n_{k}$} & \multicolumn{3}{|c|}{$d=10$} & \multicolumn{3}{|c|}{$d=100$} & \multicolumn{3}{|c|}{$d=1000$} \\
\hline & mean & 5 th & 95th & mean & 5 th & 95th & mean & 5 th & 95 th \\
\hline 2 & 0.86 & 0.40 & 1.70 & 0.24 & 0.09 & 0.60 & 0.06 & 0.01 & 0.18 \\
\hline 3 & 1.21 & 0.44 & 3.19 & 0.74 & 0.10 & 2.55 & 0.78 & 0.01 & 2.04 \\
\hline 4 & 1.04 & 0.44 & 2.52 & 0.79 & 0.11 & 2.01 & 0.73 & 0.02 & 1.80 \\
\hline 5 & 1.02 & 0.47 & 2.26 & 0.86 & 0.11 & 2.53 & 0.80 & 0.02 & 2.02 \\
\hline 10 & 1.03 & 0.53 & 1.87 & 0.91 & 0.23 & 1.74 & 0.90 & 0.19 & 1.73 \\
\hline 100 & 1.02 & 0.82 & 1.28 & 0.98 & 0.71 & 1.26 & 0.96 & 0.67 & 1.24 \\
\hline 1000 & 1.00 & 0.94 & 1.07 & 0.98 & 0.81 & 1.11 & 0.99 & 0.84 & 1.14 \\
\hline \multicolumn{10}{|c|}{$\begin{array}{c}\hat{b} \text {, estimated location parameter }(b=0) \\
\qquad d=10\end{array}$} \\
\hline$n_{k}$ & mean & 5 th & 95th & mean & 5 th & 95th & mean & 5 th & 95 th \\
\hline 2 & -0.02 & -0.96 & 1.06 & -0.30 & -1.17 & 0.36 & -0.39 & -1.23 & 0.33 \\
\hline 3 & 0.09 & -1.01 & 1.20 & -0.13 & -1.47 & 1.03 & -0.11 & -1.65 & 1.06 \\
\hline 4 & 0.01 & -0.92 & 1.12 & -0.11 & -1.16 & 1.02 & -0.15 & -1.23 & 0.98 \\
\hline 5 & -0.00 & -0.91 & 1.03 & -0.07 & -1.26 & 1.01 & -0.12 & -1.45 & 1.01 \\
\hline 10 & 0.00 & -0.73 & 0.80 & -0.08 & -1.00 & 0.70 & -0.09 & -1.02 & 0.71 \\
\hline 100 & -0.00 & -0.26 & 0.26 & -0.02 & -0.37 & 0.27 & -0.07 & -0.54 & 0.24 \\
\hline 1000 & -0.00 & -0.07 & 0.07 & -0.05 & -0.30 & 0.10 & -0.00 & -0.22 & 0.22 \\
\hline
\end{tabular}

$D$, the square root of the determinant of the Fisher information

\begin{tabular}{rrrrrrrrrr} 
& \multicolumn{3}{c}{$d=10$} & \multicolumn{3}{c}{$d=100$} & \multicolumn{3}{c}{$d=1000$} \\
$n_{k}$ & mean & 5 th & 95 th & mean & 5 th & 95 th & mean & 5 th & 95 th \\
2 & 1.98 & 0.22 & 4.71 & 3.28 & 0.28 & 9.77 & 6.75 & 0.34 & 27.02 \\
3 & 2.84 & 0.42 & 6.70 & 3.87 & 0.52 & 12.32 & 6.94 & 0.65 & 32.24 \\
4 & 3.76 & 0.91 & 8.70 & 4.20 & 0.87 & 12.92 & 6.80 & 0.78 & 30.65 \\
5 & 4.42 & 1.08 & 9.84 & 4.42 & 1.17 & 13.16 & 6.59 & 1.14 & 28.09 \\
10 & 7.72 & 2.96 & 15.22 & 6.81 & 2.75 & 13.10 & 7.14 & 2.76 & 12.33 \\
100 & 65.10 & 27.30 & 101.56 & 50.68 & 17.02 & 83.85 & 50.03 & 17.46 & 80.59 \\
1000 & 670.40 & 396.07 & 952.25 & 361.69 & 87.16 & 751.10 & 314.30 & 77.38 & 652.07
\end{tabular}


Table 6: Empirical probabilities of MLE non-existence for the cloglog model in initial estimation with equally spaced covariates. The simulation parameters are $d$, the length of the initial interval, $M$, the number of covariate points, and $n_{m}$, the number of measurements per each covariate point. The results are means from 10000 simulation runs.

\begin{tabular}{rrccc}
$M$ & $n_{m}$ & $d=10$ & $d=100$ & $d=1000$ \\
\hline 5 & 1 & 0.9686 & 1.0000 & 1.0000 \\
5 & 2 & 0.9084 & 1.0000 & 1.0000 \\
5 & 10 & 0.4641 & 1.0000 & 1.0000 \\
5 & 20 & 0.2165 & 1.0000 & 1.0000 \\
5 & 100 & 0.0029 & 1.0000 & 1.0000 \\
5 & 1000 & 0.0000 & 1.0000 & 1.0000 \\
10 & 1 & 0.7609 & 1.0000 & 1.0000 \\
10 & 2 & 0.4746 & 1.0000 & 1.0000 \\
10 & 5 & 0.1000 & 1.0000 & 1.0000 \\
10 & 10 & 0.0058 & 0.9998 & 1.0000 \\
10 & 50 & 0.0000 & 1.0000 & 1.0000 \\
10 & 500 & 0.0000 & 0.9955 & 1.0000 \\
50 & 1 & 0.0164 & 0.9407 & 1.0000 \\
50 & 2 & 0.0000 & 0.8354 & 1.0000 \\
50 & 20 & 0.0000 & 0.0756 & 1.0000 \\
100 & 1 & 0.0000 & 0.7148 & 1.0000 \\
100 & 2 & 0.0000 & 0.4014 & 1.0000 \\
100 & 10 & 0.0000 & 0.0038 & 0.9997 \\
1000 & 1 & 0.0000 & 0.0003 & 0.7070 \\
1000 & 2 & 0.0000 & 0.0000 & 0.4024
\end{tabular}


Table 7: Summary of estimated models (8).

\begin{tabular}{lcc} 
logit & \multicolumn{2}{c}{$R^{2}=0.9613$} \\
& estimate & standard error \\
Intercept $\alpha$ & -0.964 & 0.069 \\
$\log d \beta$ & -0.200 & 0.013 \\
$\log C_{S} \gamma$ & 0.839 & 0.012 \\
& \multicolumn{2}{c}{$R^{2}=0.9765$} \\
probit & estimate & standard error \\
\multicolumn{1}{c}{-1.27} & 0.058 \\
Intercept $\alpha$ & -0.223 & 0.011 \\
$\log d \beta$ & 0.892 & 0.010 \\
$\log C_{S} \gamma$ & \multicolumn{2}{c}{$R^{2}=0.9753$} \\
\multicolumn{1}{c}{$\operatorname{loglog}$} & estimate & standard error \\
& -1.063 & 0.057 \\
Intercept $\alpha$ & -0.214 & 0.010 \\
$\log d \beta$ & 0.850 & 0.010 \\
$\log C_{S} \gamma$ & \multicolumn{2}{c}{}
\end{tabular}

